\title{
Expression of HER Receptor Proteins in Prostate Adenocarcinoma: A Perspective from North Central Nigeria
}

\author{
0. I. Ajetunmobi $\left.{ }^{*}{ }^{(}\right)$, U. Drebber ${ }^{2}$, R. Buettner ${ }^{2}$, O. D. Dzuachii ${ }^{1}$, \\ R. A. Vhritherire ${ }^{3}$, 0. A. Oguntunde ${ }^{4}$, O. S. Raphael ${ }^{5}$, O. Odujoko $^{6}$ \\ ${ }^{1}$ Department of Histopathology, Federal Medical Centre Makurdi, Benue State, Nigeria \\ ${ }^{2}$ Institute of Pathology, University Hospital Cologne, Cologne, Germany \\ ${ }^{3}$ Department of Histopathology, Benue State University Teaching Hospital, Makurdi, Benue State, Nigeria \\ ${ }^{4}$ Department of Histopathology, College of Medicine, University of Lagos, Lagos, Nigeria \\ ${ }^{5}$ Department of Histopathology, University of Abuja Teaching Hospital, Abuja, Nigeria \\ ${ }^{6}$ Department of Morbid Anatomy and Forensic Medicine, Obafemi Awolowo University, Osun State, Nigeria \\ Email: *lanreolorire@gmail.com
}

How to cite this paper: Ajetunmobi, O.I., Drebber, U., Buettner, R., Dzuachii, O.D., Vhritherire, R.A., Oguntunde, O.A., Raphael, O.S. and Odujoko, O. (2019) Expression of HER Receptor Proteins in Prostate Adenocarcinoma; A Perspective from North Central Nigeria. Open Journal of Pathology, 9, 58-70. https://doi.org/10.4236/ojpathology.2019.9 $\underline{3007}$

Received: May 19, 2019

Accepted: July 23, 2019

Published: July 26, 2019

Copyright (c) 2019 by author(s) and Scientific Research Publishing Inc. This work is licensed under the Creative Commons Attribution International License (CC BY 4.0).

http://creativecommons.org/licenses/by/4.0/

\section{(c) (i) Open Access}

\begin{abstract}
Introduction: Members of the Human Epidermal Receptor [HER] family of receptor tyrosine kinases, such as HER2 and EGFR proteins are overexpressed in several epithelial malignancies and serve as effective therapeutic targets in cancer management. However, their role in prostate cancer development has been sparingly explored and with contrasting findings. Notably their relationship with prostate cancers cases seen in Sub-Saharan Africa is yet to be explored. Design: A retrospective study involving histologically diagnosed cases of adenocarcinomas of the prostate. Cases were classed according to the WHO/ISUP Gleason Prognostic groups [G1 - G5]. Immunohistochemical analysis was performed using monoclonal antibodies for HER2 and EGFR, while in situ hybridization employed DNA probes for the corresponding genes. Scores of +2 and +3 were regarded as positive for both antibodies, while a target gene: centromere ratio of $>2$ was set as the threshold for amplification. Results: A total of 44 cases were included in the study. The acinar type was the commonest morphologically, with Gleason group 5 [Gleason scores 8 - 10] accounting for close to half of the cases [47.7\%]. The HER2 antibody stained negatively in the majority of cases [93.2\%], being positive in only 3 [6.8\%] of cases seen. High level expression of EGFR $[+2 /+3]$ was observed in $25 \%$ of cases, low level expression was identified in 6 [13.6\%] cases. All HER2 positive malignancies displayed overexpression of EGFR. In situ-hybridization revealed the absence of high level amplification for both HER2 and EGFR, while polysomy was not detected in any of the cases. Con-
\end{abstract}


clusion: The overexpression of EGFR in prostate cancers has been demonstrated in a native African population, affirming its suitability for targeted therapy. Overexpression of HER2 in prostate cancer is inconstant, and amplification of the HER2 gene is less frequent than as compared to malignancies of the Breast and Ovary. There's a need for a standardized protocol for assessing HER2 in prostate cancer.

\section{Keywords}

Prostate, Adenocarcinoma, Gleason, HER2, EGFR2, Amplification, FISH

\section{Introduction}

The HER receptor family of receptor tyrosine kinases [RTK] includes proteins such as Epidermal Growth factor Receptor [EGFR], HER2/c-erbB2, HER3, and HER4. They represent the most extensively studied group of the RTKs especially with regards to their roles in normal mammalian development, physiology and formation of human cancers. Following ligand binding, these receptors undergo conformational changes such as dimerization and autophosphorylation their intrinsic tyrosine kinase residues. These events activate downstream intracellular signaling pathways such as the Ras/Raf/Mitogen-activated protein kinase (MAPK), the Phosphatidylinositol 3 kinase (PI3K)-Akt, PLC $\gamma / \mathrm{PKC}$, STAT and recently the Par6-atypical pathways. These pathways subsequently trigger transcription of numerous genes influencing major cell processes such as cell proliferation, survival and neoplastic transformation [1] [2] [3] (Figure 1).

Notably, EGFR and HER2 are overexpressed in many solid tumors, and their co-expression leads to an additive/super-additive effect. Their activation is by ligand binding in the physiologic state, however, molecular aberrations could lead to ligand independent activation. Notably dysregulation of these receptor proteins has been implicated in several epithelial and non-epithelial malignancies. Gene amplification of HER2 has been found in up to a third of breast cancers, while amplification of the EGFR gene is found in $40 \%$ of Glioblastoma multiforme [GBM] and 5\% - 10\% of non-small cell lung carcinomas [NSCLCs] [1] [2]. Overactivity and overexpression of these proteins have also been implicated in the formation of malignancies of the Skin, Genitourinary tract, gastrointestinal tract, Head and Neck and Prostate. Our grasp of their complex function and molecular regulatory networks has fuelled the development of revolutionary therapeutic options in the management of cancers over the last decade [4].

Pharmacologic entities inhibiting these receptor pathways have proven to be effective methods of providing targeted therapy in a number of malignancies. The HER2/neu protein has been demonstrated to be an effective target in molecular therapy of metastatic cancers of the breast and stomach, via the use of monoclonal antibodies [e.g. Transtuzumab] specific for the Her-2/neu protein [4] [5]. Similarly, the use of monoclonal antibodies such as Cetuximab and the 


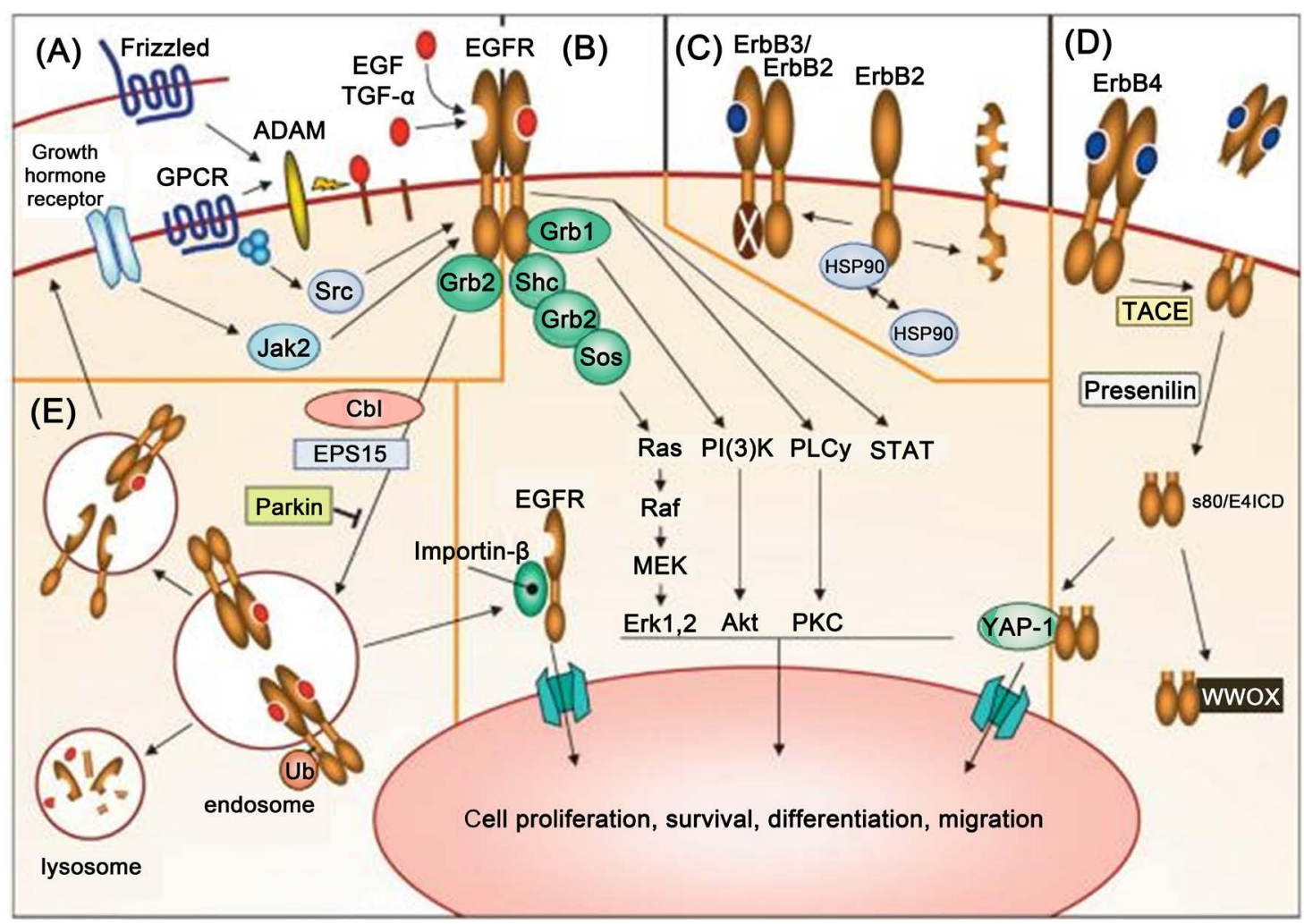

Figure 1. Schematic representation of the HER family and downstream pathways.

tyrosine kinase inhibitors, Gefitinib and Erlotinib, targeting EGFR has been rewarding in the treatment of non-small cell lung cancer, as well as cancers of the colon, pancreas and the prostate [6].

Prostate cancer [Pca] is the $2^{\text {nd }}$ commonest cause of malignancy related death in males globally. Its incidence is increasing worldwide and has attained the status of a disease of public health importance, being the most frequently diagnosed cancer in men worldwide [7] [8]. The sub-Saharan African region is accounting for increasing proportion of new cases, rising from $56 \%$ in 2008 , to $70 \%$ by 2030 [9]. Records from the two institutional cancer registries in Makurdi, North central Nigeria, infer that the prostate is the number one site of malignancies in males within the region. As a localized pathology, treatment can be easily implemented using surgery and radiotherapy, however progression to advanced stage/grade of disease transforms PCa into life-threatening, refractory illness. Despite benefiting from advances in therapeutic approaches employing Surgery, Chemotherapy, Radiotherapy, and Hormone therapy, mortality remains high accounting for more than 300,000 deaths annually across the globe. One key reason for this is the genomic complexity/heterogeneity of this malignancy as well as the programmed progression from an androgen dependent phase (AD-PCa) to an androgen-independent phase (AI-PCa) over time. Effective therapeutic options are few and far between in this hormone refractory state, which has a median survival of less than 12 months [9] [10].

In the search for novel therapies for advanced prostate cancer, potential over- 
expression of HER family of oncogenes has attracted significant interest over the last decade. The significance of these family of oncogenes and corresponding oncoproteins to prostate cancer [PCa] and their potential impact on chemotherapy have been sparingly explored and when so, with conflicting outcomes. Lyne et al. observed overexpression of the Her-2/neu protein in $82 \%$ of the 24 PCas studied, while overexpression was seen in only $36 \%$ of PCas assessed by Sadasivan [11] [12]. As early as the 90s, studies at the Mayo Clinic demonstrated increased expression of this oncogene in Prostatic Intraepithelial Neoplasm [PIN] while several studies have correlated over-expression of her2-neu with advanced grades and stages of PCa. Increased expression of this oncogene is equally correlated with metastatic disease [7] [13].

In a recent study by Schlomm et al. involving 2497 prostate tumors, an analysis of DNA and protein showed detectable expression of EGFR in $18 \%$ of cancers. This expression was associated with high Gleason grades and advanced stages. However, in another study, EGFR amplification and mutation were analysed in 10 patients with PCa and no significant correlation was found between the presence of mutations and the hormone sensitivity status. Notably, several studies have demonstrated that both Her-2/neu and EGFR activate the Androgen Receptor $[\mathrm{AR}]$ in neoplastic prostate epithelial cells via the Akt pathway. This restores AR signalling following androgen ablation, thus increasing chances of recurrence or relapse [5] [14]. Globally, about a third of newly diagnosed PCa cases present with metastases, while most cases of PCas present late in our environment, reducing chances of improved survival despite treatment [15] [16]. In the face of an increased search for effective targeted cancer therapy suitable for use in low-resource settings, exploring the pattern of expression of the HER group of oncogenes provides an opportunity to assess the potency of monoclonal antibodies against it even in advanced cases. Furthermore inasmuch as numerous studies have been performed regarding the links between HER receptor tyrosine kinases and Prostate Cancer, none have been performed in an indigenous Black African population.

This was a multi-institutional study analysing the expression the Her-2/neu and EGFR oncogenes in Prostate Cancers diagnosed at the Pathology Departments of the Federal Medical Center Makurdi and Benue State University Teaching Hospital. It assessed the relationships between the [Gleason] grades of these malignancies and the molecular expression of both the Her-2/neu and EGFR genes and protein products, while in theory exploring the suitability of monoclonal antibodies targeting these molecules in advanced PCas.

\section{Materials and Methods}

A total of 63 consecutive cases of histologically diagnosed adenocarcinomas of the prostate were identified from surgical pathology registers of the Federal Medical Center and Benue State University Teaching Hospital, both in Makurdi, North Central Nigeria, between January 2014 and June 2018. Availability of rep- 
resentative archival glass slides and Formalin Fixed Paraffin Embedded [FFPE] blocks were applied as inclusion criteria, hence only 44 of these cases were selected for the study.

Data regarding age and Gleason score were obtained from the cancer registries and surgical pathology records of these institutions. Routine Hematoxylin and Eosin slides were reviewed by two pathologists (A.O.I.) and (D.D.) to affirm the presence of primary malignancy, assign Gleason scores while also documenting other histologic features such as morphologic type, perineural infiltration, and vascular invasion.

Ethical approval was obtained from the Institutional Review Board of the Federal Medical Centre, Makurdi.

\subsection{Immunohistochemical Analysis}

Fresh serial sections 3 - $5 \mu \mathrm{m}$ thick were made for immunohistochemical staining which was performed using the following monoclonal antibodies; Her2-neu [Ventana/Roche, Pre-diluted, 4B5 Rabbit] and Epidermal Growth Factor Receptor/EGFR [Dako, clone 2-18C9, pre-diluted]. The polymer detection method of the indirect immunoperoxidase technique was utilized. The Leica Bond Max and Ventana Medical Systems Autostainers were used. The steps for deparaffinization, dehydration, antigen retrieval and incubation with monoclonal antibodies were performed according to manufacturers' instructions using the above mentioned autostainers. The sites of immunoprecipitate formation were identified using a Carl Zeiss light microscope [Scope A1]. Intensity and distribution of Her2-neu staining were scored from 0 to $3+$ and cytoplasmic/membranous respectively. Staining for EGFR was reported as positive or negative and scored from 0 to $3+$ in terms of intensity and proportion of tumor cells positive. Negative and positive controls included breast cancer sections with known levels of HER2 expression and Skin for the EGFR. The procedures for immunostaining were carried out at the Institute fur Pathologie of the University Hospital Cologne, Germany. Results were depicted using frequency tables, pie and bar charts.

\subsection{Tissue Micro Array}

Tissue microarrays were constructed for the purpose of conducting FISH analysis on the samples. A total of 32 biopsies were included in the arrays, 16 being excluded for technical reasons such as core size and diameter of neoplastic focus. Using the Hematoxylin and Eosin slides, the areas with the most characteristic pathologic features were marked and were used to delineate the corresponding areas on the donor tissue blocks. A Beecher Instrument array was used to make punches $0.6 \mathrm{~mm}$ in diameter in the donor blocks. These donor cores were then transferred to a recipient tissue block constructed using an empty paraffin block.

\subsection{Fluorescent in Situ Hybridization}

Fluorescent in Situ Hybridization (FISH) was performed using the protocol of 
the Institute fur Pathologie, University Hospital Cologne. Briefly, $2 \mu \mathrm{m}$ thick sections of FFPE, in a micro-array, were passed through a pre-treatment solution, deparaffinised and dehydrated using the VP2000 machine set at $75^{\circ} \mathrm{C}$. Two gene probes for detecting amplifications in the EGFR and Her2 genes (ZytoLight Spec EGFR/CEN7 and Zytolight Spec Her2/CEN17 respectively) were applied to the two appropriately labelled sections of the micro-array. Hybridization was performed in a Dako Cytomatic Hybridizer at a temperature of $37^{\circ} \mathrm{C}$ over a period of $12-14 \mathrm{hrs}$. A post hybridization buffer $2 \mathrm{xSSC}$ was applied to the micro-array, placed in a water bath at 56C for a total of 6 minutes. Following this, the DiaminoBenzidene chromogene was applied. The slides were viewed using a Leica DM 5500 B Fluorescent microscope utilizing oil immersion.

\section{Results}

A total of 44 cases of Prostate cancer were included in this study. The mean age was $64.9 \pm 11.8 \mathrm{yrs}$, the median was $63 \mathrm{yrs}$, while the peak age of incidence/diagnosis was between 51 and 60 yrs. There were no cases diagnosed before the age of 40 , as the age range was $44-95$ yrs.

The commonest histologic type was the acinar type accounting for 38 (86.4\%) of samples assessed, the ductal type comprising only $2(4.5 \%)$ and a combined acinar-ductal morphology observed in 4 (9.1\%). For this analysis, the cancers were divided into 5 groups [G1 - G5] according to their Gleason scores. A large proportion of the cases $(21[47.7 \%])$ were high grade, having Gleason scores of 8 - 10 and thus falling into Gleason groups G4 and G5. Gleason group 1 i.e. Gleason score $\leq 6$ accounted for $11(25 \%)$ of cases while groups 2 and 3 accounted for $8(18.2 \%)$ and $4(9.1 \%)$ cases respectively. Table 1 Patients aged 70 and above had a mean Gleason score of 7.3 with $40 \%$ being High grade, while patients younger than 70 yrs had a mean Gleason score of $7.6 \%$ with $50 \%$ exhibiting high grade morphology.

Overexpression of EGFR was defined by immunostaining having either of these characteristic patterns; +2 positive staining in $\geq 50 \%$ of tumor cells OR +3 staining intensity in $\geq 30 \%$ of tumor cell population. This was observed in 11 cases (25\%). Low level overexpression of EGFR was defined as +1 staining and this was observed in $6(13.6 \%)$ cases, thus a total of 17 (38.6\%) cases were positive for EGFR in varying intensities. Notably, a solitary case displayed positive nuclear staining in contrast to the conventional/accepted membranous and cytoplasmic staining patterns. The Her2neu antibody stained negative in the majority of cases 41 (93.2\%), being only positive in $3(6.8 \%)$ cases, of the positive cases, 2 (4.5\%) showed cytoplasmic staining, while only 1 (2.3\%) displayed positive membranous staining. Figure 2 All Her2 positive cases displayed high level of EGFR expression.

Notably EGFR expression was imperceptible in most of the high grade cancers, being observed in only 5 (23.8\%) of this group of Prostate cancers. Positive EGFR staining was higher in lower grade tumors with G1 and G2 malignancies 
Table 1. Summary of patient characteristics.

\begin{tabular}{|c|c|c|}
\hline Variable & Frequency & Percentage \\
\hline \multicolumn{3}{|l|}{ Age Range } \\
\hline$<40$ & 0 & 0 \\
\hline $41-50$ & 3 & 6.8 \\
\hline $51-60$ & 15 & 34.1 \\
\hline $61-70$ & 12 & 27.3 \\
\hline $71-80$ & 9 & 20.4 \\
\hline$>80$ & 4 & 9.1 \\
\hline Not stated & 1 & 2.3 \\
\hline Total & 44 & 100 \\
\hline \multicolumn{3}{|l|}{ Histologic type } \\
\hline Acinar & 38 & 86.4 \\
\hline Ductal & 2 & 4.5 \\
\hline Mixed & 4 & 9.1 \\
\hline Total & 44 & 100 \\
\hline \multicolumn{3}{|l|}{ Gleason Group } \\
\hline G1 $(\leq 6)$ & 11 & 25.0 \\
\hline $\mathrm{G} 2(3+4)$ & 8 & 18.2 \\
\hline $\mathrm{G} 3(4+3)$ & 4 & 9.1 \\
\hline G4 (8) & 4 & 9.1 \\
\hline G5 (>8) & 17 & 38.6 \\
\hline Total & 44 & 100 \\
\hline \multicolumn{3}{|l|}{ Specimen type } \\
\hline Prostatectomies & 3 & 6.8 \\
\hline Needle biopsies & 41 & 93.2 \\
\hline Total & 44 & 100 \\
\hline
\end{tabular}

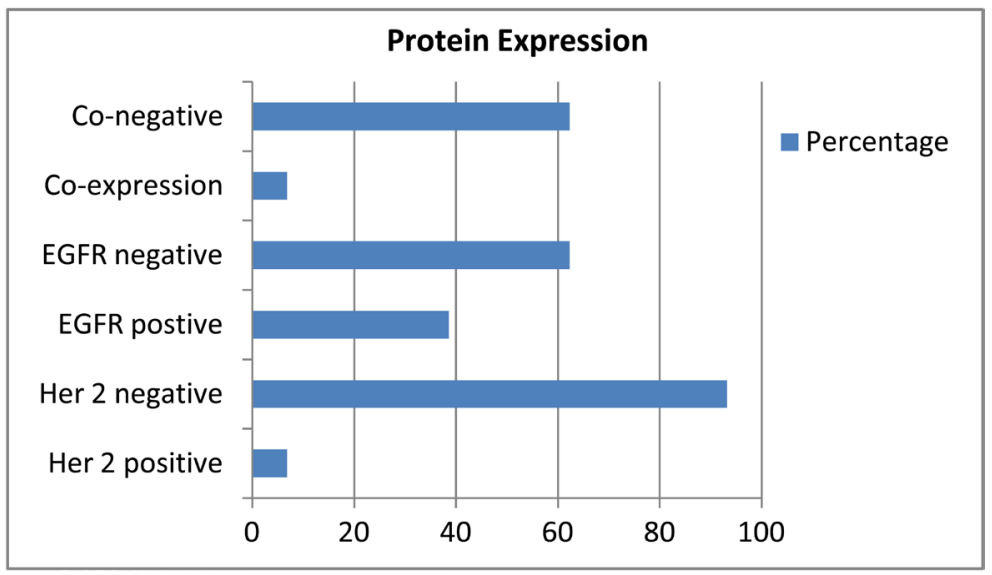

Figure 2. Expression patterns of EGFR and HER2 on immunohistochemistry.

showing EGFR positivity of $54.5 \%$ and $62.5 \%$ respectively. Sections containing adjacent areas of normal and hyperplastic prostatic acini showed strong positive staining in basal and luminal cells, whereas areas of malignant acini showed 
comparatively weaker expression of EGFR. Figure 3 All sections showing predominant ductal or mixed acinar-ductal morphology were negative for both EGFR and Her2 on immunohistochemistry.

A total of 32 samples were subjected to gene analysis by Fluorescent in situ Hybridization, and of these, only 16 gave perceptible signals for EGFR and only 12 for Her2-neu. None of these samples showed high level amplification of either the Her2 neu or EGFR genes. Figure 4 However, for Her2, low level amplification (defined as Her2/centromere 17 ratio of 1.5 - 2.5) was observed in two samples. Polysomy was not observed in any of the FISH sections examined.

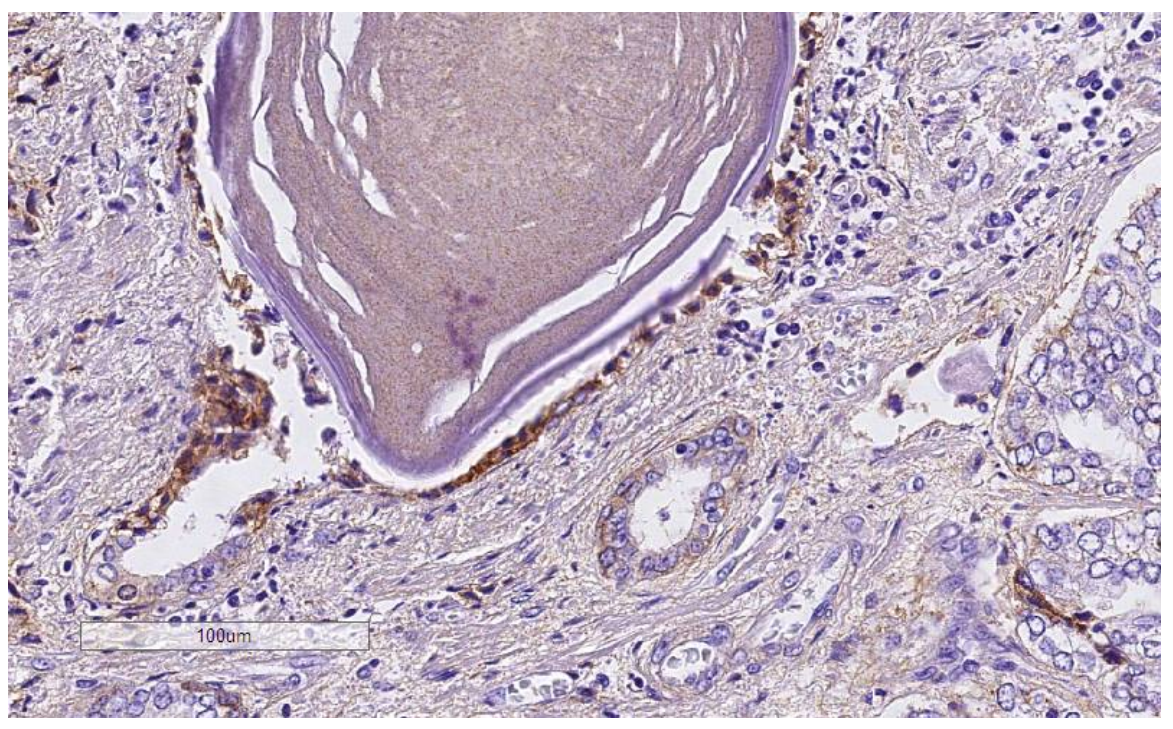

Figure 3. Differential expression of EGFR between adjacent neoplastic and hyperplastic prostatic acini.

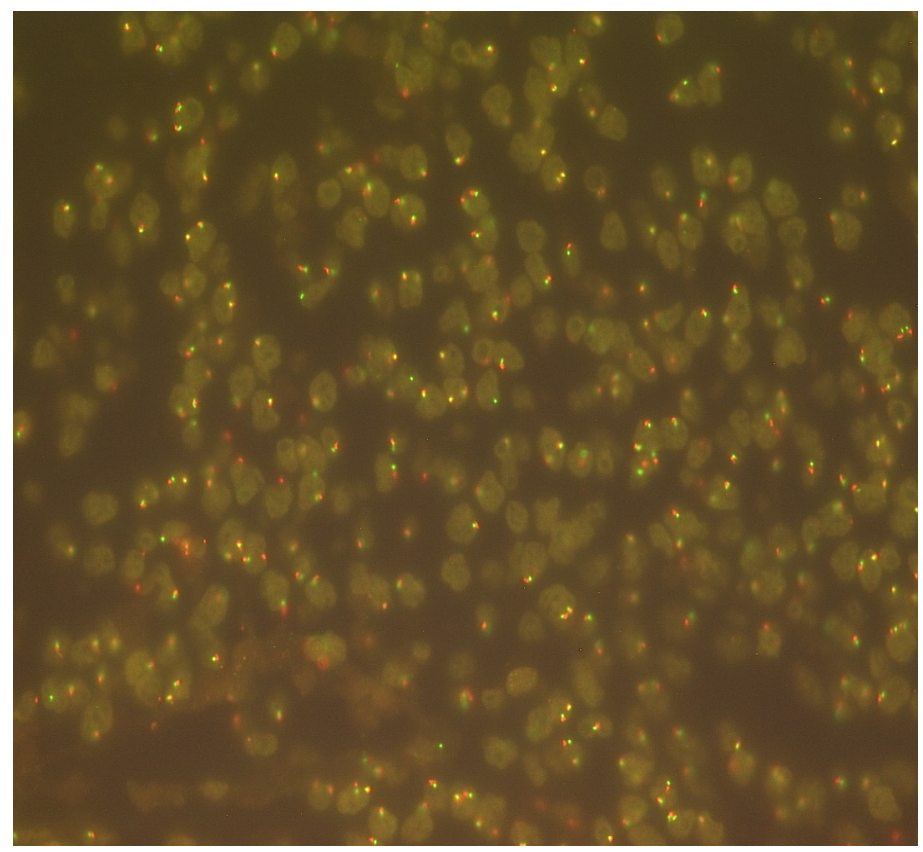

Figure 4. FISH slide showing absence of gene amplification. 


\section{Discussion}

The age distribution in this study reflects findings in the United States and Egypt, where the average age of diagnosis was 66 yrs and 67.38 yrs respectively. However, in the United Kingdom the peak age range is in the $>90$ age group, in contrast to our observation of $51-60$ yrs. In line with observations in the above referenced studies, cases of Prostate Cancer were rare before the age of 40 yrs [17] [18] [19].

Pure ductal CA is a rare morphologic variant of prostate adenocarcinoma. Global estimates range from $0.5 \%$ to $6 \%$ of cases, and it is mostly seen in the elderly. Observations from this index study show that 2 cases (4.5\%) of PCas were ductal and were seen at ages 44 and 57 [20].

In recent times, the World Health Organization (WHO) has adopted a 5 tiered division of Gleason scores into prognostic classes (Table 1). In a study in Egypt, $56.7 \%$ of PCa cases were high grade, with Gleason scores between 8 and 10 , while $32.0 \%$ and $36.7 \%$ of cases were high grade in studies carried out in Ukraine and Lagos, Southwest Nigeria respectively [18] [21] [22]. Our observation of $47.7 \%$ falls into an intermediate position. In sharp contrast, $80 \%$ of prostatectomy cases in Iran had Gleason scores less than 7. It should be noted that there is an inherent tendency for higher grades in needle biopsies as sampling errors might result in an underestimation or overestimation of the Gleason grade [23]. Assessment of biochemical relapse, diagnosis of Androgen Independence and pathologic staging all present with significant logistic difficulties, as most patients present late OR are suboptimal for localized treatment i.e. radical prostatectomy with radiotherapy. Hence most diagnoses of PCa in Nigeria/Sub-Saharan Africa are made on needle biopsies [9].

The rate of EGFR overexpression in this study (25\%), was lower than observations in Italy (36\%) and Iran 59\%, but higher than findings in Hamburg (18\%). Furthermore, while there was an inverse relationship between EGFR expression and grade in this study, no correlation was seen between these parameters in Iran. In Italy however, 13\% of cases displayed mutations in the EGFR Tyrosine Kinase domain, (similar to those found in patients with NSCLC) and all of these 13 cases had Gleason scores $\geq 7$. The absence of Gene amplification in our study reflects findings in Hamburg, where only $0.25 \%$ of the 2446 cases showed amplification of the EGFR gene. However, low level gene copy number gains were observed in this study. Several studies have shown association between such low level gene copy number gains with positive IHC expression [14] [23] [24].

It is postulated that genetic factors affect EGFR expression on IHC. Some studies have demonstrated reduced expression of the receptor protein with advancing malignant behaviour while other reports have documented equivalent expression of EGFR in both benign and malignant prostate lesions. Incidentally, a mutant form of the EGFR \{EGFRvIII\} has been demonstrated to be expressed selectively in $\mathrm{PCa}$ and is indicative of an aggressive prostate cancer phenotype [25] [26]. These variable findings reflect two key issues; 1) Accurate definition of 
EGFR status is vital in prognostic stratification of PCa pts and selection for targeted therapy; 2) The need for larger, multi institutional studies assessing these oncogenes at the DNA, RNA and protein levels.

The exact prevalence of Her2 protein overexpression and gene amplification in Primary PCa remains unclear. In concordance with our findings, researchers at Indiana, in the United States, reported that (Using the standard method), only 1 of 38 cases demonstrated Her2 overexpression while none of the 38 samples displayed Her2 amplification on FISH [7]. Equally 0 of the 147 samples analysed by Visakorpi et al. displayed expression of Her2. However, Ross documented gene amplification in $41 \%$ of PCas studied [27] [28] [29] [30]. The likely reason for this variation is a lack of standardized methodology. Most if not all studies assessing Her2 expression in prostate, have employed antibodies and protocols validated for use on other organs such as Breast and Stomach. Some studies have even used polyclonal antibodies, while others have considered cytoplasmic staining as positive. Highly reproducible, organ specific, validated techniques are pivotal, to ensure comparable data across various studies. Another plausible hypothesis is that since Her2 has no known ligand, its association with EGFR cell signalling is linked to its ability to form heterodimers with other members of the HER protein family. This "secondary recruitment" might account for its inconsistent expression [7] [10] [26].

Furthermore, the pattern of Her2 expression appears to change as Prostate cancer evolves from an androgen dependent to an androgen independent state. Experiments using human Prostate cancer cell lines showed increased expression in androgen independent states. This is believed to be due to selective growth and survival of Her2 expressing cells which normally do not require androgens. Regardless of the aforementioned, a number of key studies have established the fact that Her2 is involved in the development of Prostate cancer, being also implicated in increasing invasiveness and facilitating vascular dissemination [11] [31] [32].

Several types of immunotherapies directed againstHer2 have shown promising clinical results, equally patients vaccinated with a HER2 polypeptide vaccine (AE37) showed improved overall survival. Immunization against specific oncogenes, represent an exciting prospect in personalized medicine and effective application could translate to life transforming results in oncology [32].

\section{Limitations}

The relative absence of demonstrable amplification of the selected oncogenes in this study is plausibly largely due to technical factors in the pre-analytical and analytical phases of the preparation of $\mathrm{H} \& \mathrm{E}$ sections. Data from the Biospecimen Research Network documented that, for FISH, whole sections were preferable to needle cores and ischemia time should not exceed $1 \mathrm{hr}$ [33]. It cannot be guaranteed that these conditions were met for all specimens involved in this study. Inasmuch as rapid technological advancement is being made in molecular 
biology techniques, the process of preparing FFPEs is still central to achieving quality results with most procedures. In the same vein, tumor heterogeneity alongside the use of TMA on core needle biopsies would produce sampling errors, contributing to suboptimal results.

In conclusion, this is the first prostate cancer study to report the pattern of the expressions of EGFR and Her2 in a black African population, at both the level of the surface protein and the corresponding genes. Further studies including a larger number of cases, with a wider geographic spread across sub-Saharan Africa and data on biochemical relapse, are advocated. The identification of new reliable biomarkers that can predict progression to hormone refractoriness and serve as therapeutic targets is both urgent and essential.

\section{Funding}

This project was funded by a Technical Transfer Fellowship awarded by the International Union for Cancer Control [UICC]. Additional funding was provided by the Institute of Pathology, University Hospital, Cologne.

\section{Acknowledgements}

The authors acknowledge the valuable assistance of Professor Alexander Quass of the Institute of Pathology in Cologne, as well as the technical staff of the Pathology laboratories in Federal Medical Centre Makurdi and the University Hospital Cologne.

\section{Conflicts of Interest}

The authors have no conflicts to declare

\section{References}

[1] Wieduwilt, M.J. and Moasser, M.M. (2008) The Epidermal Growth Factor Receptor Family: Biology Driving Targeted Therapeutics. Cellular and Molecular Life Sciences, 65, 1566-1584. https://doi.org/10.1007/s00018-008-7440-8

[2] Arteaga, C.L. and Engelman, J.A. (2014) ERBB Receptors: From Oncogene Discovery to Basic Science to Mechanism-Based Cancer Therapeutics. Cancer Cell, 25, 282-303. https://doi.org/10.1016/j.ccr.2014.02.025

[3] Dhomen, N.S., Mariadason, J., Tebbutt, N. and Scott, A.M. (2012) Therapeutic Targeting of the Epidermal Growth Factor Receptor in Human Cancer. Critical Reviews in Oncogenesis, 17, 31-50. https://doi.org/10.1615/CritRevOncog.v17.i1.40

[4] Nelson, E.L. (2014) HER2/Neu: An Increasingly Important Therapeutic Target: Part 2 Distribution of HER2/Neu Overexpression and Gene Amplification by Organ, Tumour Site and Histology. Journal of Clinical Investigation (London), 4, 705-728. https://doi.org/10.4155/cli.14.62

[5] Wen, Y., Hu, M.C.T., Makino, K., Spohn, B., Bartholomeusz, G., Yan, D.H., et al. (2000) HER-2/Neu Promotes Androgen Independent Survival and Growth of Prostate Cancer Cells via the Akt Pathway. Cancer Research, 60, 6841-6845.

[6] Guérin, O., Fischel, J.L., Ferrero, J.-M., Bozec, A. and Milano, G. (2010) EGFR Targeting in Hormone-Refractory Prostate Cancer: Current Appraisal and Prospects 
for Treatment. Pharmaceuticals, 3, 2238-2247. https://doi.org/10.3390/ph3072238

[7] Sanchez, K.M., Sweeney, C.J., Mass, R., Koch, M.O., Eckert, G.J., Geary, W.A., et al. (2002) Evaluation of HER-2/Neu Expression in Prostatic Adenocarcinoma: A Request for a Standardized Organ Specific Methodology. Cancer, 95, 1650-1655.

https://doi.org/10.1002/cncr.10839

[8] Ayala, G., Frolov, A., Chatterjee, D., He, D., Hilsenbeck, S. and Ittmann, M. (2015) Expression of ERG Protein in Prostate Cancer: Variability and Biological Correlates. Endocrine-Related Cancer, 22, 277-287. https://doi.org/10.1530/ERC-14-0586

[9] Adeloye, D., David, R.A., Aderemi, A.V., Iseolorunkanmi, A., Oyedokun, A., Iweala, E.E.J., et al. (2016) An Estimate of the Incidence of Prostate Cancer in Africa: A Systematic Review and Meta-Analysis. PLoS ONE, 11, e0153496. https://doi.org/10.1371/journal.pone.0153496

[10] Ullen, A., Lennartsson, L., Harmenberg, U., Lennernas, B., Majumuder, K., Holmberg, A.R., et al. (2005) Prostate Cancer Cell Lines Lack Amplification: Overexpression of HER2. Acta Oncologica, 44, 490-495. https://doi.org/10.1080/02841860510029888

[11] Lyne, J.C., Naraghi, R.M., Melhem, M.A., et al. (1995) Human Prostate Cancer Cells Respond to But Do Not Express Neu Differentiation Factor (NDF) in Spite of HER2/Neu Gene Amplification and Protein Overexpression. The Cancer Journal from Scientific American, 3, 21-30.

[12] Sadasivan, R., Morgan, R., Jennings, S., Austenfeld, M., Van Veldhuizen, P., Stephens, R., et al. (1993) Overexpression of Her-2/Neu May Be an Indicator of Poor Prognosis in Prostate Cancer. Journal of Urology, 150, 126-131. https://doi.org/10.1016/S0022-5347(17)35413-7

[13] Botswick, D.G. (1994) c-erbB-2 Oncogene Expression in Prostate Intraepithelial Neoplasia. Journal of the National Cancer Institute, 86, 1108-1110. https://doi.org/10.1093/jnci/86.15.1108

[14] Niyat, M.M. (2015) Prognostic Value of Her2/Neu Expression in Patients with Prostate Cancer: A Systematic Review. Reviews in Clinical Medicine, 2, 168-173.

[15] Chen, Y., Lin, Y., Nie, P., Jiang, W., Liu, Y., Yuan, R., et al. (2017) Associations of Prostate-Specific Antigen, Prostate Carcinoma Tissue Gleason Score, and Androgen Receptor Expression with Bone Metastasis in Patients with Prostate Carcinoma. Medical Science Monitor, 23, 1768-1774. https://doi.org/10.12659/MSM.900977

[16] American Cancer Society (2019) Key Statistics for Prostate Cancer. http://www.cancer.org

[17] Daoud, S.A., Darweesh, M.F. and Sharaf, R.M. (2016) HER2/Neu Immunohistochemical Overexpression in Prostatic Adenocarcinoma in Relation to Tumor Pathological Behavior. Middle East Journal of Scientific Research, 24, 459-464.

[18] Cancer Research UK. Prostate Cancer Statistics. http://www.cancerresearchuk.org

[19] Tkachenko, P.V. (2017) Clinical-Morphological Prognostic Characteristics of Prostate Cancer. German Science Herald, 2, 14-18.

[20] Dube, V.E., Farrow, G.M. and Greene, L.F. (1973) Prostatic Adenocarcinoma of Ductal Origin. Cancer, 32, 402-409. https://doi.org/10.1002/1097-0142(197308)32:2<402::AID-CNCR2820320218>3.0.C O;2-Q

[21] Odubanjo, M.O., Banjo, A.A.F., Ayoola, S., Abdulkareem, F.B., Anunobi, C.C. and Olayinka, A.A. (2013) The Clincopathologic Pattern of Prostate Carcinoma in La- 
gos, Nigeria. North American Journal of Medical Sciences, 6, 71-75.

[22] Mofid, B., Jalali Nadoushan, M.R., Rakhsha, A., Mirzaei, H.R. and Zeinali, L. (2010) Epidermal Growth Factor Receptor Gene in Prostate Cancer after Radical Prostatectomy. Iranian Journal of Cancer Prevention, 3, 174-177.

[23] Schlomm, T., Kirtsein, P., Iwers, L., Daniel, B., Steuber, T., Walz, J., et al. (2007) Clinical Significance of Epidermal Growth factor Receptor Overexpression and Gene Copy Number Gains in Prostate Cancer. Clinical Cancer Research, 13, 6579-6584. https://doi.org/10.1158/1078-0432.CCR-07-1257

[24] Peraldo-Neia, C., Migliardi, G., Mello-Grand, M., Montumerro, F., Segir, R., Pignochino, Y., et al. (2011) Epidermal Growth Factor Receptor (EGFR) Mutation Analysis, Gene Expression Profiling and EGFR Protein Expression in Primary Prostate Cancer. BMC Cancer, 11, 31. https://doi.org/10.1186/1471-2407-11-31

[25] Olapade-Olaopa, E.O., Moscatello, D.K., MacKay, E.H., Horsburgh, T., Sandhu, D.P.S., Terry, T.R., et al. (2000) Evidence for the Differential Expression of a Variant EGF Receptor Protein in Human Prostate Cancer. British Journal of Cancer, 82, 186-194. https://doi.org/10.1054/bjoc. 1999.0898

[26] Ratan, H.L., Gescher, A., Steward, W.P. and Mellon, J.K. (2003) ErbB Receptors: Possible Therapeutic Targets in Prostate Cancer? BJU International, 92, 890-895. https://doi.org/10.1111/j.1464-410X.2003.04503.x

[27] Minner, S., Jessen, B., Stiedenroth, L., Burandt, E., Kollerman, J., Mirlacher, M., et al. (2010) Low Level HER2 Overexpression Is Associated with Rapid Tumor Cell Proliferation and Poor Prognosis in Prostate Cancer. Clinical Cancer Research, 16, 1553-1560. https://doi.org/10.1158/1078-0432.CCR-09-2546

[28] Visakorpi, T., Kallioniemi, O.P., Koivula, T., Harvey, J. and Isola, J. (1992) Expression of Epidermal Growth Factor Receptor and ERBB2 (HER-2/Neu) Oncoprotein in Prostatic Carcinomas. Modern Pathology, 5, 643-648.

[29] Kaltz-Wittmer, C., Klenk, U., Glaessgen, A., et al. (2000) FISH Analysis of Gene Aberrations (MYC, CCND1, ERBB2, RB, and AR) in Advanced Prostatic Carcinoma before and after Androgen Deprivation Therapy. Laboratory Investigation, 80, 1455-1464. https://doi.org/10.1038/labinvest.3780152

[30] Ross, J.S., Nazeer, T., Church, K., Amato, C., Figge, H., Rifkin, M.D. and Fisher, H.A. (1993) Contribution of HER-2/Neu Oncogene Expression to Tumor Grade and DNA Content Analysis in the Prediction of Prostatic Carcinoma Metastasis. Cancer, 72, 3020-3028.

https://doi.org/10.1002/1097-0142(19931115)72:10<3020::AID-CNCR2820721026> 3.0.CO;2-\#

[31] Da Cruz, J.A.S., dos Reis, S.T., Guariero, M.E.S., Leite, K.R., Srougi, M. and Passerotti, C.C. (2017) Association between HER-2/Neu(erbB-2) Overexpression and Biochemical Recurrence of Prostate Cancer after Radical Prostatectomy. Annals of Urology Research, 1, 1006.

[32] Oxley, J.D., Winkler, M.H., Gillatt, D.A. and Peat, D.S. (2002) Her2/Neu Oncogene Amplification in Clinically Localized Prostate Cancer. Journal of Clinical Pathology, 55, 118-120. https://doi.org/10.1136/jcp.55.2.118

[33] Bass, B.P., Engel, K.B., Greytak, S.R. and Moore, H.M. (2014) A Review of Preanalytical Factors Affecting Molecular, Protein, and Morphological Analysis of Formalin-Fixed, Paraffin-Embedded (FFPE) Tissue: How Well Do You Know Your FFPE Specimen? Archives of Pathology \& Laboratory Medicine, 138, 1520-1530. https://doi.org/10.5858/arpa.2013-0691-RA 